\title{
ON THE INDEPENDENCE OF NAMING AND LOCATING MASKED TARGETS IN VISUAL SEARCH*
}

\author{
GORDON D. LOGAN $†$ \\ McGill University
}

\begin{abstract}
AASTRACT
Twelve Ss were required to name or locate masked letters in arrays containing noise elements. Accuracy in locating was lower when target letters were presented among Vs than when presented among triangles or with no noise. Naming was as accurate when Vs and triangles were presented with the target as when no noise was presented. Since noise did not affect naming in the same way it affected locating, it was concluded that naming is independent of locating.
\end{abstract}

Recently, Baron (1973) AND SNyder (1972) sugcested that the target in visual search tasks must be located before it can be named; thus naming is thought to be contingent on locating. The experiment reported here tested two predictions derived from this hypothesis.

The first prediction was based on the contention that the encoding of a character's location must occur prior to the encoding of its identity. If this is true, locating responses should be more accurate than naming responses. This prediction is readily confirmed in studies where single letters are presented (Dick \& Dick, 1969; Logan, in press), but when noise items are simultaneously present in the array, a different picture emerges. When the target and noise are easy to discriminate, locating is more accurate than naming, but when the discrimination is difficult, locating and naming are equally accurate (Logan, in press).

In terms of a strong interpretation of the first prediction (i.e., that locating must be more accurate than naming in all conditions), these latter results speak against the notion of contingent processing. However, a weaker interpretation is possible: the prediction may merely serve to differentiate conditions where processing is contingent from those where it is not. Because of this ambiguity, the second prediction was derived.

The notion of a contingency between locating and naming implies that manipulations which impair locating accuracy should produce a similar

'This research was supported in part by Grant no. A0127 from the National Research Council of Canada to Albert S. Bregman. The author was supported by a postgraduate scholarship from the National Research Council of Canada. The experiment was reported at the annual meeting of the Canadian Psychological Association, June 1974, Windsor, Ontario. The author is grateful to Jane Zbrodoff and Barry O'Neil for their help in the information analyses.

TRequests for reprints should be sent to Gordon D. Logan, Department of Psychology, Queen's University, Kingston, Ontario, Canada.

Canad. J. Psychol./Rev. Canad. Psychol., 1975, 29 (1) 
pattern of impairment in naming accuracy. A different pattern of impairment (or no impairment) would support the notion that locating and naming are independent. The advantage of this prediction is that, unlike the first, it is only meaningful when all conditions are considered together (i.e., it has no weak interpretation).

The experiment was essentially a replication of Logan's (in press) conditions in a within-subject design. Subjects were required to name or locate single target letters in a backward masking paradigm. The targets were either presented alone, or with triangles or Vs serving as noise items, simultaneously present in the array.

\section{Method}

\section{Subjects}

Twelve McGill students, 10 female and 2 male, served as paid Ss. All had normal or corrected vision and none made errors on unmasked trials during practice.

\section{Apparatus and Stimuli}

The stimuli were presented binocularly in a Gerbrands three-field tachistoscope (Model T-3B-1). The target letter was a lowercase a, $o$, $e$, or $c$ appearing in one of the four corners of an imaginary square centred on the fixation point. Depending on the noise condition, the other three positions were blank, contained lowercase Vs or solid triangles made by filling in inverted lowercase Vs. The masks were made from crosses superimposed on capital $O$ 's (i.e. $\oplus$ ). One such mask was centred on each corner of the imaginary square around the fixation point so that a target letter (and each noise element) presented simultaneously would be imbedded in one of the masks. Black Letraset 36 pt Futura Medium letters were mounted on white cards to make the targets and mask. Viewed at a distance of $80 \mathrm{~cm}$, each target letter and noise element subtended about $26^{\prime} \times 26^{\prime}$ of visual angle and each mask in the masking stimulus subtended about $43^{\prime} \times 43^{\prime}$ of visual angle. The distance between the corners of the imaginary square on which both the target letters and the masks were centred was about $2^{\circ}$ of visual angle.

The target letters were exposed for $10 \mathrm{msec}$ and the masking stimulus was exposed for $25 \mathrm{msec}$, both at a luminance of approximately $8 \mathrm{ftL}$. Between trials and during interstimulus intervals, a fixation field was exposed. It contained a small white dot in the centre of a black field, presented at a luminance below the lower limit of the measuring device (an SEI spot photometre). Interstimulus interval was varied from 0 to $100 \mathrm{msec}$ in $25 \mathrm{msec}$ steps. On one-sixth of the trials, no mask was presented. During testing, the room was dimly lit by a 40 -watt bulb. Each day, at least 5 min were allowed for dark adaptation before testing began.

\section{Procedure}

On each trial, Ss were required to do one of three tasks: location where Ss reported the location of the target letter without prior knowledge of its name, name where $\mathrm{Ss}$ reported the name of the target letter without prior. knowledge of its location, and name-given-location where Ss reported the name of the target letter, having been told its location prior to stimulus presentation. This last task was included to assess the importance of selective attention in name processing. If selective attention is 
important, providing a cue for selection should increase accuracy relative to the name task. In all tasks, forced-choice responding was required.

Every $S$ completed each task in each of three noise conditions (no noise, Vs noise, triangles noise). The tasks were run in blocks of 96 trials in which each combination of four letters ( $a, 0, e$, and $c$ ), four locations (top left, top right, bottom left, and bottom right), and six interstimulus intervals $(0,25,50,75,100 \mathrm{msec}$, and no mask) occurred once. Two random orders of the 96 trials were used in alternate blocks throughout the experiment and the order of alternation was counterbalanced across Ss. Each $S$ completed two blocks a day for five days. The first was practice and included blocks of each combination of noise condition and task. Following practice, each $S$ began with one noise condition and completed one block of each task before proceeding to the next. Three such orders were derived from the combination of separate Latin squares for noise and tasks such that element $(i, j)$ in the combined square contained element $(i, j)$ of the noise square and row (i) of the task square. Four Ss were assigned to each order.

On the first day, Ss were shown examples of each type of stimulus and stimulus sequence (mask versus no mask) and the three tasks were explained to them. On each day, Ss were told the sequence of tasks and noise conditions before testing began. Ss were instructed to maintain rigid fixation on the fixation point before and during each trial. Every trial began with a verbal ready signal from $E$ to which $S$ responded affirmatively if he had the fixation point in clear focus: About $0.5 \mathrm{sec}$ after $S$ indicated his readiness, $E$ initiated the stimulus sequence and the target letter appeared immediately.

\section{RESULTS}

In each task in each noise condition, each $S$ had 16 forced-choice trials at each interstimulus interval. To correct for guessing, $4 / 3$ of the errors were subtracted from 16 . These scores were submitted to analysis of variance. Separate three-way analyses were carried out for each task (Ss $\times$ noise $\times$ interstimulus interval) and each noise condition (Ss $\times$ task $\times$ interstimulus interval).

\section{Tasks}

The effect of noise on the tasks is of primary interest and can be summarized as follows: noise affected locating but not naming or naminggiven-location. The crucial differences between the results of the naming and location tasks can be seen in Figures 1 and 2. Table I contains the data from the name-given-location task.

This pattern of differences is supported by the analyses of variance. In all analyses, the main effect of interstimulus interval was highly significant. When another main effect was significant, it usually interacted significantly with interstimulus interval, indicating a ceiling effect at the longer interstimulus intervals.

The main effect of noise was significant in the locating task, $F(2,22)=$ $17.74, p<.01$. Target letters were located as accurately in the context of 


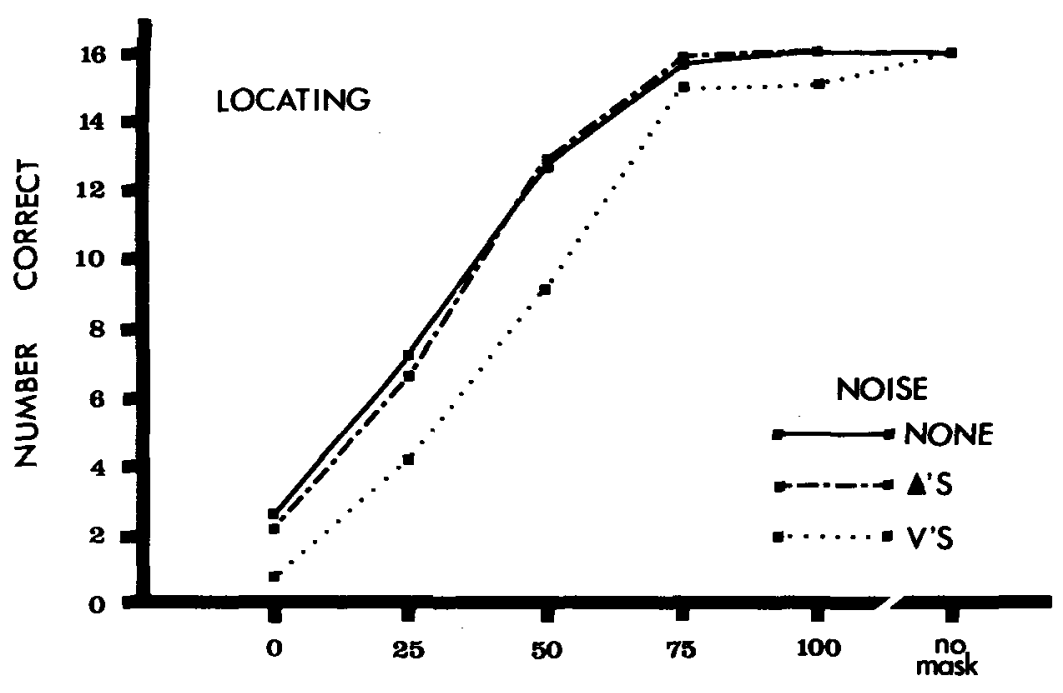

INTERSTIMULUS INTERVAL

IN MSEC

Fig. 1. Mean number of correct responses in the location task, corrected for guessing, as a function of noise condition and interstimulus interval.

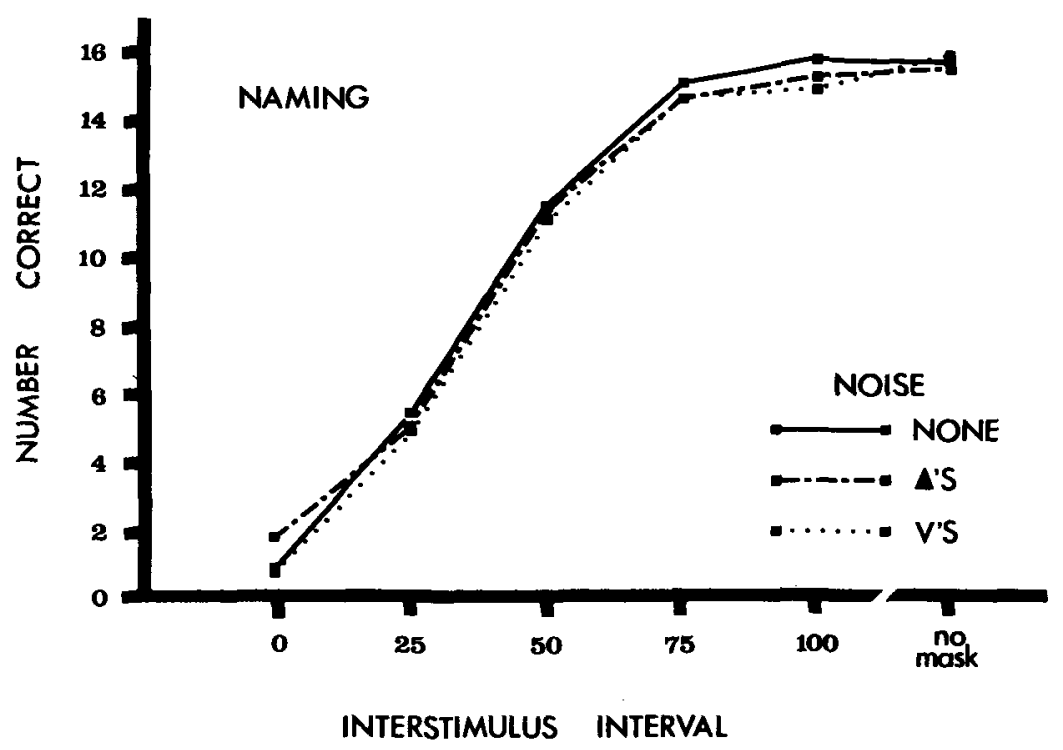

IN MSEC

Fic. 2. Mean number of correct responses in the name task, corrected for guessing, as a function of noise condition and interstimulus interval. 
simultaneously present triangles as with no noise, $t(22)\langle 1, p\rangle .05$, but less accurately in the context of simultaneously presented Vs, $t(22)=6.98$, $p<.01$. No such differences were observed in the naming task or in the naming-given-location task. In both analyses, the main effect of noise was not significant, $F \mathrm{~s}(2,22)=0.46$ and 0.42 , respectively, $p>.05$.

\section{Noise Conditions}

The effects of tasks within noise conditions are also of interest and can be summarized as follows: within each noise condition, the name-given-location task was superior to the name task. With no noise and with triangles as noise, locating was as accurate as naming-given-location, but with Vs as noise, locating accuracy dropped to the level of performance in the name task. These effects can be seen in Table I where the rows represent tasks and noise conditions and the columns represent interstimulus intervals. Again, these conclusions are supported by the analyses of variance and again, interstimulus interval was highly significant and usually interacted with other significant main effects.

TABLE I

MEAN NUMBER OF CORRECT RESPONSES, CORRECTED FOR GUESSING, IN TASKS AND NOISE CONDITIONS AS A FUNCTION OF INTERSTIMULUS INTERVAL

\begin{tabular}{|c|c|c|c|c|c|c|c|}
\hline \multirow{2}{*}{ Noise condition } & \multirow[b]{2}{*}{ Task } & \multicolumn{6}{|c|}{ Interstimulus interval in msec } \\
\hline & & $\mathbf{0}$ & 25 & 50 & 75 & 100 & no mask \\
\hline No noise & $\underset{\mathbf{N} / \mathbf{L}}{\mathbf{L}}$ & $\begin{array}{l}2.67 \\
1.00 \\
0.83\end{array}$ & $\begin{array}{l}7.25 \\
5.42 \\
8.75\end{array}$ & $\begin{array}{l}12.75 \\
11.58 \\
12.75\end{array}$ & $\begin{array}{l}15.67 \\
15.08 \\
15.25\end{array}$ & $\begin{array}{l}16.00 \\
15.83 \\
15.67\end{array}$ & $\begin{array}{l}16.00 \\
15.75 \\
15.92\end{array}$ \\
\hline Triangles noise & $\underset{\mathbf{N} \mid \mathbf{L}}{\stackrel{\mathbf{L}}{\mathbf{N}}}$ & $\begin{array}{l}2.25 \\
1.92 \\
1.83\end{array}$ & $\begin{array}{l}6.67 \\
5.17 \\
8.17\end{array}$ & $\begin{array}{l}12.83 \\
11.50 \\
12.83\end{array}$ & $\begin{array}{l}15.75 \\
14.67 \\
15.50\end{array}$ & $\begin{array}{l}16.00 \\
15.33 \\
15.67\end{array}$ & $\begin{array}{l}16.00 \\
15.58 \\
15.92\end{array}$ \\
\hline Vs noise & $\underset{\mathbf{N} \mid \mathbf{L}}{\mathbf{L}}$ & $\begin{array}{l}0.83 \\
0.91 \\
0.25\end{array}$ & $\begin{array}{l}4.25 \\
4.92 \\
8.08\end{array}$ & $\begin{array}{r}9.08 \\
11.08 \\
13.25\end{array}$ & $\begin{array}{l}14.92 \\
14.67 \\
15.17\end{array}$ & $\begin{array}{l}15.00 \\
14.92 \\
15.58\end{array}$ & $\begin{array}{l}16.00 \\
15.92 \\
15.83\end{array}$ \\
\hline
\end{tabular}

The main effect of tasks was significant with no noise, $F(2,22)=4.65$, $p<.05$. Here locating and naming-given-location were more accurate than naming, $t$ s $(22)=4.10$ and 3.26 , respectively, $p<.01$. With triangles as noise, the main effect of tasks was not significant, $F(2,22)=3.32, p>.05$, but subsequent $t$ tests showed locating and naming-given-location to be significantly more accurate than naming, $t$ s $(22)=3.05$ and 3.29 , respectively, $p<.01$. The main effect of tasks was significant with Vs as noise, $F(2,22)=9.42, p<.01$, and as in the previous noise conditions, naminggiven-location was more accurate than naming, $t(22)=4.26, p<.01$. Unlike the previous noise conditions, locating was not significantly different from naming, $t(22)=1.73, p>.05$. 
TABLE II

MEAN INFORMATION TRANSMITTED IN BITS IN TASKS AND NOISE CONDITIONS

(the maximum information transmitted is 2 bits)

\begin{tabular}{lccc}
\hline & \multicolumn{3}{c}{ Noise condition } \\
\cline { 2 - 4 } Task & No noise & Triangles noise & Vs noise \\
\hline L & 1.045 & 0.942 & 0.783 \\
N & 0.902 & 0.904 & 0.849 \\
N $\mid$ L & 1.006 & 1.060 & 0.997 \\
\hline
\end{tabular}

Since accuracy scores in 4-alternative forced-choice situations are not sensitive to response biases or confusions among stimuli, it was considered desirable to supplement the accuracy data with an analysis of information transmitted (Garner, 1962). Stimulus-response matrices were derived for each $S$ in each condition and task. To provide a sufficient number of observations in each matrix, like cases were collapsed across interstimulus interval. Table II contains the mean (across Ss) amount of information transmitted in each task and condition. Five of $12 \mathrm{Ss}$ transmitted more location information with no noise than with triangles as noise. This difference was not significant by a sign test. Eleven Ss transmitted more location information with triangles than with Vs as noise $(p<.02$ by sign test). Further, no significant differences in information transmitted were obtained in the naming task. Eight of $12 \mathrm{Ss}$ transmitted more name information with triangles than with no noise, and 7 of 12 transmitted more with no noise than with Vs. These results confirm the conclusions drawn from the accuracy data.

When the locating and naming tasks are compared, a different picture emerges. Here, none of the differences were significant by a sign test, though 8 of 12 Ss transmitted more information about location than name with no noise, 9 of 12 did so with triangles as noise, and 5 of 12 did so with Vs as noise.

To summarize the important results, presenting noise items simultaneously with the target decreased locating performance only when the noise items were Vs. No such effects were observed with triangles as noise. Noise had no effect on either naming task. Comparing tasks within noise conditions, naming-given-location was consistently superior to naming. What changed as noise changed was the relation between the locating task and the naming tasks. With no noise and with triangles as noise, locating accuracy was as good as naming-given-location, but with Vs as noise, locating accuracy dropped to the level of the name task. However, these comparisons between tasks should be interpreted with caution since they were not corroborated by the information analyses. 


\section{Discussion}

The present experiment demonstrated that accuracy in locating can be impaired with no effect on the accuracy of naming. Further, while locating accuracy was higher than naming accuracy most of the time, this effect was not consistently observed across noise conditions, nor was it apparent in the information analyses. Since naming and locating are uncorrelated in this way, it seems fair to conclude that they are independent (Garner \& Morton, 1969). However, the question remains as to whether the processes underlying these responses are independent as well. The answer depends on the degree of correspondence between the spatial coding used in the visual system and that used in subject's responses.

For the purposes of discussion, let us call locating within the visual system locating, and locating in terms of response co-ordinates locating . $_{1}$ Consider the case where locating 1 and locating ${ }_{2}$ do not correspond. From this point of view, locating ${ }_{1}$ serves to organize the encoding of the items in the array and locating 2 is a result of the encoding. In the present experiment, locating ${ }_{1}$ would be equally accurate in each task and noise condition,

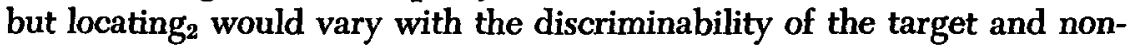
target items. This conception accounts nicely for the difference in locating 2 performance between the Vs noise and triangles noise conditions. The triangles differ from the targets in terms of brightness and category (letter versus geometric form), and so discriminating between them should be easier than discriminating Vs from the targets, where no brightness or category differences exist. Thus locating, performance should be better with triangles than with Vs as noise, and it was.

However, this conception predicts a difference in locating 2 performance between the no-noise and triangles-noise conditions. On the average, more encoding would have to go on for a locating 2 response to occur with triangles as noise. Locating ${ }_{1}$ would be sufficient to enable a locating 2 response with no noise, since only the target item was presented, but with triangles as noise locating ${ }_{1}$ would specify four items, and some additional encoding would be necessary to discriminate the target. Such encoding would take time and produce lower locating 2 performance with triangles as noise than with no noise. No such differences were apparent in the accuracy data or in the information analyses.

Now, consider the case where locating ${ }_{1}$ and locating 2 do correspond. If locating $_{1}$ is sensitive to brightness differences, all of the locating ${ }_{2}$ results can be explained. Targets in the no-noise and triangles-noise conditions can be specified by their brightness; no-noise targets are darker than their background, while triangles-noise targets are lighter. To the extent that these brightness differences are equal, though opposite in sign, locat- 
ing $_{2}$ performance should be equal in the two conditions. Admittedly, the differences were not scaled in the present experiment, but accuracy and information measures are consistent with this interpretation.

Since targets and noise are equal in brightness (again unscaled) with Vs as noise, locating ${ }_{1}$ cannot separate the target from the noise. More encoding - perhaps naming the target - would be necessary for them to be discriminated. Once this has occurred, the location $_{1}$ of the target can be determined, enabling a locating ${ }_{2}$ response. Performance here would not be as good as the other noise conditions, because more encoding is required, and it was not. Note that from this point of view the additional encoding does not involve locating or locating. $_{2}$.

To the extent that the reader finds these arguments reasonable, it can be concluded that locating and naming processes function independently. This conclusion is consistent with the finding that name and location information decay independently in sensory memory (Dick, 1969; Townsend, 1973). Indeed, independent decay would be expected, given the present results.

\section{RÉSỪÉ}

Douze sujets ont à nommer ou à localiser des lettres masquées dans des ensembles contenant des éléments de bruit. Les résultats montrent que la localisation est moins exacte quand les lettres cibles sont présentées parmi des $\mathrm{V}$ plutôt que parmi des triangles ou en l'absence de bruit. L'identification des lettres est aussi exacte quand des $V$ et des triangles sont présentés avec la cible que quand aucun bruit n'est présent. Le fait que le bruit n'affecte pas l'identification comme il affecte la localisation permet de conclure que l'identification et la localisation sont indépendantes.

\section{REFERENCES}

Baron, J. Perceptual dependence: evidence for an internal threshold. Percept. \& Psychophys., 1973, 13, 527-533.

Dick, A.O. Relations between the sensory register and short-term storage in tachistoscopic recognition. J. exp. Psychol., 1969, 82, 279-284.

Dick, A.O., \& Dick, S.O. An analysis of hierarchical processing in visual perception. Canad. J. Psychol., 1969, 23, 203-211.

GARNER, W.R. Uncertainty and structure as psychological concepts. New York: Wiley, 1962.

Garner, W.R., \& Morton, J. Perceptual independence: definitions, models, and experimental paradigms. Psychol. Bull., 1969, 72, 233-259.

LOGAN, G.D. On the relation between identifying and locating masked targets in visual search. Quart. J. exp. Psychol. In press.

SNYDER, C.R.R. Selection, inspection and naming in visual search. J. exp. Psychol., $1972,92,428-431$.

TownSEND, V.M. Loss of spatial and identity information following a tachistoscopic exposure. J. exp. Psychol., 1973, 98, 113-118. 